\title{
Criminologie
}

\section{Mieux saisir les outils actuariels : une analyse factorielle de la Statique-99R}

\author{
Sébastien Brouillette-Alarie, Jean Proulx et Massil Benbouriche
}

Volume 46, numéro 1, printemps 2013

URI : https://id.erudit.org/iderudit/1015299ar

DOI : https://doi.org/10.7202/1015299ar

Aller au sommaire du numéro

Éditeur(s)

Les Presses de l’Université de Montréal

ISSN

0316-0041 (imprimé)

1492-1367 (numérique)

Découvrir la revue

Citer cet article

Brouillette-Alarie, S., Proulx, J. \& Benbouriche, M. (2013). Mieux saisir les outils actuariels : une analyse factorielle de la Statique-99R. Criminologie, 46(1),

199-219. https://doi.org/10.7202/1015299ar
Résumé de l'article

Une critique couramment formulée à l'encontre des outils actuariels statiques concerne leur incapacité à éclairer l'intervention, au regard de leurs fondements athéoriques. Dans la cadre d'un effort visant à nuancer cette critique, la présente étude a tenté d'identifier les construits théoriques présents dans la Statique-99R, l'échelle actuarielle de prédiction de la récidive des délinquants sexuels la plus utilisée. Une analyse factorielle exploratoire des items de l'instrument en a extrait trois facteurs : la délinquance sexuelle, la délinquance générale et le jeune âge. Une interprétation de ces facteurs en ce qui a trait aux dimensions psychologiques a été proposée : la délinquance sexuelle en tant qu'indicatrice de déviance sexuelle ou de préoccupations sexuelles et la délinquance générale en tant qu'indicatrice de traits antisociaux et/ou de psychopathie. Les limites et les implications pour de futures recherches sont discutées. 


\title{
Mieux saisir les outils actuariels : une analyse factorielle de la Statique-99R
}

\author{
Sébastien Brouillette-Alarie \\ Université de Montréal, Institut Philippe-Pinel de Montréal \\ sebastien.brouillette-alarie@umontreal.ca \\ Jean Proulx \\ Université de Montréal, Institut Philippe-Pinel de Montréal \\ jean.proulx@umontreal.ca

\section{Massil Benbouriche} \\ Université de Montréal, Université européenne de Bretagne - Rennes 2 \\ Laboratoire Applications de la réalité virtuelle en psychiatrie légale \\ massil.benbouriche@umontreal.ca
}

\begin{abstract}
RÉSUMÉ • Une critique couramment formulée à l'encontre des outils actuariels statiques concerne leur incapacité à éclairer l'intervention, au regard de leurs fondements athéoriques. Dans la cadre d'un effort visant à nuancer cette critique, la présente étude a tenté d'identifier les construits théoriques présents dans la Statique-99R, l'échelle actuarielle de prédiction de la récidive des délinquants sexuels la plus utilisée. Une analyse factorielle exploratoire des items de l'instrument en a extrait trois facteurs: la délinquance sexuelle, la délinquance générale et le jeune âge. Une interprétation de ces facteurs en ce qui a trait aux dimensions psychologiques a été proposée: la délinquance sexuelle en tant qu'indicatrice de déviance sexuelle ou de préoccupations sexuelles et la délinquance générale en tant qu'indicatrice de traits antisociaux et/ou de psychopathie. Les limites et les implications pour de futures recherches sont discutées.
\end{abstract}

MOTS-CLÉs - Agresseurs sexuels, facteurs de risque statiques, facteurs de risque dynamiques, Statique-99, analyse factorielle, dimensions psychologiques. 


\section{Introduction ${ }^{1}$}

\section{Contexte historique}

Les méthodes utilisées pour prédire la récidive criminelle ont connu une évolution importante depuis 50 ans. Le jugement clinique non structuré a progressivement laissé place à l'évaluation actuarielle, une méthode d'évaluation du risque basée sur une combinaison mécanique de facteurs de risque empiriquement validés. En Amérique du Nord, la prédiction actuarielle a été massivement adoptée, ayant démontré à maintes reprises la supériorité de sa validité prédictive sur le jugement clinique non structuré (Meehl, 1954; Dawes et al., 1989; Mossman, 1994; Gardner et al., 1996; Grove et al., 2000; Guay, 2006; Quinsey et al., 2006; Hanson et Morton-Bourgon, 2009).

Les facteurs de risque évalués par les outils actuariels peuvent être de nature statique ou dynamique. Les facteurs statiques sont des éléments d'anamnèse qui ne peuvent changer après une intervention, tandis que les facteurs dynamiques sont des caractéristiques modifiables d'un délinquant qui, lorsque réduites, s'accompagnent d'une baisse du niveau de risque de récidive (Cortoni, 2009). Les facteurs dynamiques permettraient ainsi de tenir compte des variations du niveau de risque (c.-à-d. du changement) et d'identifier des cibles de traitement (Hanson et Harris, 1998; Thornton, 2002; Quinsey et al., 2006; Cortoni, 2009).

Dans le champ de la délinquance sexuelle, les échelles actuarielles les plus fréquemment utilisées sont le Minnesota Sex Offender Screening Tool - Revised (MnSOST-R, Epperson et al., 1998), le Violence Risk Appraisal Guide (VRAG, Harris et al., 1993), le Sex Offender Risk Appraisal Guide (SORAG, Quinsey et al., 2006), le Rapid Risk Assessment for Sex Offense Recidivism (RRASOR, Hanson, 1997) et la Statique-99 (Hanson et Thornton, 2000; Hanson et Morton-Bourgon, 2004). Bien que certains de ces instruments incorporent des facteurs dynamiques, la majorité des facteurs évalués par lesdits instruments sont statiques. La Statique-99, le plus populaire d'entre eux (Archer et al., 2006; Jackson et Hess, 2007; McGrath et al., 2010), est, tel que l'indique son nom, exclusivement composée de facteurs statiques. L'utilisation exclusive de facteurs de risque

1. Note des auteurs: Nous tenons à remercier R. Karl Hanson et Leslie Helmus pour leurs données et conseils. Ce projet a été financé par le Conseil de recherches en sciences humaines du Canada. Toute question ou commentaire concernant cet article doit être acheminé à Sébastien Brouillette-Alarie, École de criminologie, Université de Montréal, C. P. 6128, succursale Centre-ville, Montréal, Québec, Canada, H3C 3J7. 
statiques présenterait plusieurs inconvénients, notamment l'incapacité à identifier des cibles thérapeutiques et à mesurer le changement, de même que l'incapacité à comprendre la dynamique causale menant à la récidive (Gendreau et al., 1996; Cortoni, 2009). Bien que leur validité prédictive ne soit pas en deçà des échelles actuarielles dynamiques (Beech et al., 2002; Knight et Thornton, 2007), les échelles purement statiques ne répondraient pas au besoin qu'ont les évaluateurs de comprendre les mécanismes générateurs de risque chez leurs clients (Mann et al., 2010). Par exemple, le fait qu'un détenu ait déjà agressé sexuellement un enfant masculin est en soi moins éclairant que d'être au fait de ses fantaisies sexuelles pédophiliques susceptibles de le vulnérabiliser relativement à un éventuel passage à l'acte.

\section{Enjeux}

Certains auteurs considèrent néanmoins que la distinction entre les facteurs de risque statiques et dynamiques est essentiellement superficielle (Beech et Ward, 2004; Mann et al., 2010). Selon ces derniers, les facteurs statiques ne sont que l'expression comportementale de dimensions (ou mécanismes) psychologiques typiquement désignées comme des facteurs dynamiques. Par exemple, un long historique de délits à caractère sexuel - un facteur statique - pourrait être compris comme l'actualisation d'intérêts sexuels déviants - un facteur dynamique. Parallèlement, n'avoir jamais entretenu de relations intimes de longue durée pourrait être le reflet d'un manque d'habiletés sociales ou, à l'inverse, de schèmes relationnels égocentriques centrés sur la conquête. Si un tel cadre conceptuel permet de mieux comprendre la signification des facteurs de risque statiques, il est néanmoins primordial de comprendre précisément à quels facteurs dynamiques/mécanismes psychologiques réfère chacun de ces facteurs statiques. La présente étude s'inscrit dans ce cadre conceptuel et vise à identifier les dimensions psychologiques présentes dans l'échelle actuarielle la plus utilisée (Archer et al., 2006; Jackson et Hess, 2007 ; McGrath et al., 2010), la Statique-99.

\section{La Statique-99}

La Statique-99 (Hanson et Thornton, 2000) est une échelle composite basée sur le RRASOR (Hanson, 1997) et le Structured Anchored Clinical Judgement (SACJ, Grubin, 1998), et regroupe les meilleurs prédicteurs 
statiques de la récidive sexuelle et violente des délinquants sexuels masculins adultes. C'est une échelle additive associant chaque facteur de risque à un score; un score total élevé rend alors compte d'un risque de récidive plus important. Bien que l'utilisation de l'échelle demande une formation, sa cotation est rapide et aisée, participant à sa popularité. Cela ne nuit aucunement à sa validité prédictive, considérée comme l'une des meilleures du domaine (Barbaree et al., 2001; Parent et al., 2011). La Statique-99 contient 10 items, détaillés dans le tableau 1.

TABLEA U 1

Items de la Statique-99

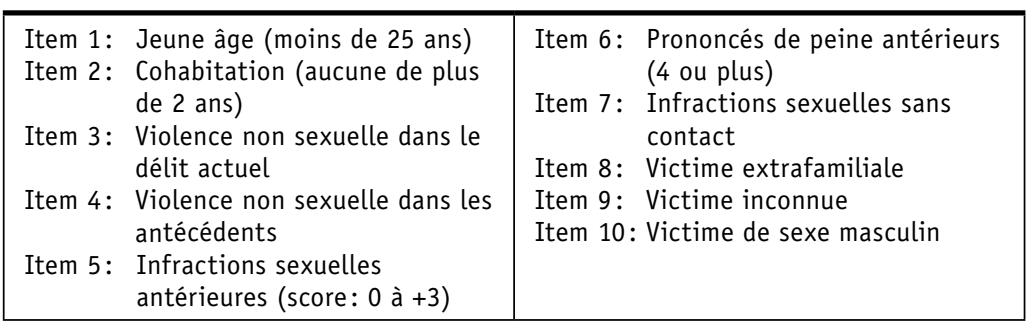

Plusieurs divisions conceptuelles de la Statique-99 ont été proposées, nous renseignant sur les dimensions psychologiques susceptibles d'y être présentes (Doren, 2004; Brouillette-Alarie et Proulx, 2008; Nunes et Cortoni, 2008). Les divisions conceptuelles de la Statique-99 sont fort cohérentes et s'entendent sur une structure à deux construits: la délinquance générale et la délinquance sexuelle, indicateurs respectifs de traits antisociaux et de déviance sexuelle (Doren, 2004). La dimension de délinquance générale/traits antisociaux serait composée d'items évaluant le jeune âge, l'absence de relation intime de plus de deux ans, les comportements de violence non sexuelle et le nombre de prononcés de peine antérieurs (Brouillette-Alarie et Proulx, 2008; Nunes et Cortoni, 2008). La deuxième dimension, la délinquance sexuelle/déviance sexuelle, serait composée des cinq autres items; soit du nombre d'infractions sexuelles antérieures, des infractions sexuelles sans contact et des items évaluant les caractéristiques des victimes sexuelles - victimes extrafamiliales, inconnues et/ou masculines (Brouillette-Alarie et Proulx, 2008; Nunes et Cortoni, 2008). 


\section{Problématique}

Bien que ces divisions conceptuelles puissent rendre compte des mécanismes psychologiques représentés dans la Statique-99, il n'en demeure pas moins qu'une preuve empirique de ces affirmations est nécessaire. Trop peu d'études empiriques se sont concentrées sur l'identification des dimensions de la Statique- $99^{2}$, et aucune d'elles n'a proposé une interprétation des dimensions identifiées relativement aux facteurs de risque dynamiques et/ou aux mécanismes psychologiques (Pham et Ducro, 2008; Walters et al., 2009). À la lumière de ces limites, la présente étude a pour buts d'identifier empiriquement les dimensions de la Statique$99 \mathrm{R}^{3}$, et d'en proposer une interprétation relativement aux dimensions psychologiques susceptibles d'améliorer la compréhension qu'ont les évaluateurs de leur outil de travail et, de fait, de la récidive sexuelle.

\section{Méthodologie}

Participants

La présente étude utilise une base de données composée de 711 délinquants sexuels masculins adultes. Ces participants provenaient de deux établissements: 1'Institut Philippe-Pinel de Montréal et le Centre régional de réception. L'Institut Philippe-Pinel est un établissement psychiatrique à sécurité maximale situé à Montréal (Québec), qui compte une unité spécialisée dans le traitement des agresseurs sexuels, tandis que le Centre régional de réception est un établissement de détention fédéral qui reçoit des délinquants ayant reçu une sentence de plus de deux ans. La base de données a été fournie par R. Karl Hanson et Leslie Helmus (Sécurité publique Canada), et contenait l'ensemble des participants des deux établissements pour lesquels nous disposions d'un formulaire de consentement.

Parmi les 711 délinquants sexuels, il y avait 352 agresseurs sexuels d'enfants, 251 agresseurs de femmes, 90 agresseurs mixtes (ayant des victimes mineures et majeures) et 18 agresseurs dont le type n'était pas spécifié. Les délinquants sexuels étaient âgés de 18 à 77 ans $(\mathrm{m}=40,85$,

2. Du moins, peu d'études ont identifié les dimensions de l'intégralité de la Statique-99.

3. Tel que mentionné dans la section méthodologie, notre étude utilise la version la plus récente de la Statique-99, soit la Statique-99R. 
é.-t. $=12,08)$ au moment où la Statique-99R a été cotée. Chaque participant ayant une valeur manquante à l'une des variables utilisées dans les analyses statistiques n'était pas retenu.

\section{Variables}

\section{Instruments}

La présente étude emploie la Statique-99R (Helmus, 2009), la dernière révision de la Statique-99 (Hanson et Thornton, 2000). L'unique différence entre les deux versions concerne l'item évaluant l'âge; au lieu d'être dichotomisé ( $0=25$ ans et plus, $1=$ moins de 25 ans), il est pondéré sur une échelle à 4 niveaux $(-3$ à +1$)$, beaucoup plus précise. Cette mise à jour permet à la Statique-99R de tenir compte du fait qu'un âge avancé réduit considérablement le risque de récidive (Hanson, 2002).

\section{Statistiques descriptives}

Le tableau 2 fournit des données descriptives pour l'ensemble des variables utilisées dans les analyses statistiques. Le score moyen de nos participants à la Statique-99R était similaire $(\mathrm{m}=2,68$, é.-t. $=2,50)$ à celui de l'échantillon méta-analytique ayant servi au développement de l'instrument ( $\mathrm{n}=7$ 491, $\mathrm{m}=2,7$, é.-t. $=2,6$, Babchishin et al., 2011). Les agresseurs de femmes adultes $(\mathrm{m}=3,51$, é.-t. $=2,09)$ avaient un score moyen à la Statique-99R plus élevé que les agresseurs d'enfants $(\mathrm{m}=2,25$, é.-t. $=2,55)$.

\section{Stratégie analytique}

L'objectif de la présente étude étant d'identifier empiriquement les dimensions présentes dans la Statique-99R, l'analyse factorielle exploratoire fut employée, étant appropriée à la réalisation d'une telle tâche. L'analyse factorielle exploratoire est une procédure statistique que l'on applique à un ensemble de variables afin d'identifier des sous-ensembles de variables cohérents et relativement indépendants les uns des autres ${ }^{4}$ (Tabachnick et Fidell, 2001). Dans le cadre de notre étude, les sousensembles de variables sont conceptualisés comme des facteurs latents

4. Communément appelés facteurs ou facteurs latents - à ne pas confondre avec facteurs de risque. 
(ou dimensions psychologiques) responsables de la manifestation conjointe de comportements (items) décrits par la Statique-99R. Les facteurs latents, n'étant pas directement observables, sont inférés au regard du patron de corrélation observé entre les items.

Les paramètres de l'analyse factorielle étaient les suivants: extraction sous forme de moindres carrés non pondérés plutôt qu'analyse en composantes principales (afin d'identifier des facteurs ayant une valeur théorique), et rotation oblique plutôt qu'orthogonale (afin que les corrélations entre nos facteurs ne soient pas artificiellement réduites) (Tabachnick et Fidell, 2001; Jöreskog, 2003 ; Costello et Osborne, 2005). Le nombre de facteurs retenus fut évalué en fonction de leur valeur propre (eigenvalue), ainsi que de l'interprétation du graphique des éboulis (scree test).

Le logiciel utilisé pour les analyses statistiques était SPSS 20.

TABLEAU 2

\section{Statistiques descriptives}

\begin{tabular}{|c|c|c|c|}
\hline Variables & $\begin{array}{l}\text { Délinquants } \\
\text { sexuels } \\
(n=711)\end{array}$ & $\begin{array}{c}\text { Agresseurs } \\
\text { d'enfants } \\
(n=352)\end{array}$ & $\begin{array}{c}\text { Agresseurs de } \\
\text { femmes } \\
(n=251)\end{array}$ \\
\hline \multicolumn{4}{|l|}{ Items de la Statique-99R } \\
\hline Âge (score: -3 à +1 ) & $-0,31$ (é.-t. = 1,17) & $-0,55$ (é.-t. = 1,15) & 0,17 (é.-t. $=0,96$ ) \\
\hline $\begin{array}{l}\text { Absence de cohabitation de } \\
\text { plus de } 2 \text { ans }\end{array}$ & $22,2 \%$ & $19,5 \%$ & $25,9 \%$ \\
\hline $\begin{array}{l}\text { Violence non sexuelle dans le } \\
\text { délit actuel }\end{array}$ & $19,0 \%$ & $14,8 \%$ & $23,1 \%$ \\
\hline $\begin{array}{l}\text { Violence non sexuelle dans } \\
\text { antécédents }\end{array}$ & $35,4 \%$ & $25,9 \%$ & $51,4 \%$ \\
\hline $\begin{array}{l}\text { Infractions sexuelles } \\
\text { antérieures (score: } 0 \text { à }+3 \text { ) }\end{array}$ & 0,65 (é.-t. = 0,99) & 0,78 (é.-t. = 1,09) & 0,52 (é.-t. $=0,86$ ) \\
\hline Prononcés de peine antérieurs & $36,3 \%$ & $29,0 \%$ & $46,2 \%$ \\
\hline $\begin{array}{l}\text { Infractions sexuelles sans } \\
\text { contact }\end{array}$ & $16,7 \%$ & $21,3 \%$ & $11,2 \%$ \\
\hline Victime sans lien de parenté & $56,4 \%$ & $42,9 \%$ & $77,7 \%$ \\
\hline Victime inconnue & $22,2 \%$ & $12,8 \%$ & $39,0 \%$ \\
\hline Victime de sexe masculin & $26,9 \%$ & $36,9 \%$ & $9,2 \%$ \\
\hline Score total à la Statique-99R & 2,68 (é.-t. = 2,50) & 2,25 (é.-t. = 2,55) & 3,51 (é.-t. $=2,09)$ \\
\hline \multicolumn{4}{|l|}{ Récidive (suivi de 5 ans) } \\
\hline Récidive sexuelle & $11,8 \%$ & $12,6 \%$ & $11,0 \%$ \\
\hline Récidive violente non sexuelle & $12,6 \%$ & $8,5 \%$ & $14,1 \%$ \\
\hline Récidive générale & $27,0 \%$ & $15,7 \%$ & $41,3 \%$ \\
\hline
\end{tabular}




\section{Résultats}

\section{Matrice de corrélations}

La matrice de corrélations inter-items utilisée par l'analyse factorielle est présentée dans le tableau 3. La matrice de corrélations n'a mis en lumière aucun cas de multicolinéarité $(r>0,80)$, indiquant qu'aucun item de la Statique-99R n'était redondant.

TABLEA U 3

Matrice des corrélations entre les items de la Statique-99R $(n=679)$

\begin{tabular}{|c|c|c|c|c|c|c|c|c|c|c|}
\hline Items & 1 & 2 & 3 & 4 & 5 & 6 & 7 & 8 & 9 & 10 \\
\hline 1 & 1 & - & - & - & - & - & - & - & - & - \\
\hline 2 & $0,35^{* * *}$ & 1 & - & - & - & - & - & - & - & - \\
\hline 3 & $0,10^{*}$ & 0,01 & 1 & - & - & - & - & - & - & - \\
\hline 4 & $0,11^{* *}$ & $-0,07$ & $0,19^{* * *}$ & 1 & - & - & - & - & - & - \\
\hline 5 & $-0,13^{* *}$ & 0,03 & $-0,02$ & $0,09^{*}$ & 1 & - & - & - & - & - \\
\hline 6 & $-0,04$ & $-0,06$ & 0,07 & $0,55^{* * *}$ & $0,24^{* * *}$ & 1 & - & - & - & - \\
\hline 7 & $-0,16^{* * *}$ & $-0,04$ & $-0,13^{* *}$ & $-0,05$ & $0,39^{* * *}$ & $0,09^{*}$ & 1 & - & - & - \\
\hline 8 & $0,22^{* * *}$ & $0,26^{* * *}$ & $-0,02$ & $0,12^{* *}$ & $0,17^{* * *}$ & $0,17^{* * *}$ & 0,06 & 1 & - & - \\
\hline 9 & $0,26^{* * *}$ & $0,23^{* * *}$ & $0,10^{*}$ & $0,09^{*}$ & $0,10^{* *}$ & $0,12^{* *}$ & 0,07 & $0,48^{* * *}$ & 1 & - \\
\hline 10 & $-0,11^{* *}$ & 0,05 & $-0,12^{* *}$ & $-0,15^{* * *}$ & $0,25^{* * *}$ & $-0,05$ & $0,19^{* * *}$ & $0,10^{* *}$ & $-0,05$ & 1 \\
\hline
\end{tabular}

* $\mathrm{p}<0,05 * * \mathrm{p}<0,01 * * * \mathrm{p}<0,001$

Item 1: âge; item 2: cohabitation (aucune de plus de 2 ans); item 3 : violence non sexuelle dans le délit actuel; item 4 : violence non sexuelle dans les antécédents; item 5 : infractions sexuelles antérieures; item 6 : prononcés de peine antérieurs ( 4 ou plus); item $7:$ infractions sexuelles sans contact; item 8: victime extrafamiliale; item 9: victime inconnue; item 10: victime de sexe masculin.

Les corrélations les plus élevées étaient entre le jeune âge et l'absence de relation intime de plus de deux ans $(r=0,35, \mathrm{p}<0,001)$, entre les antécédents de violence non sexuelle et le nombre de prononcés de peine antérieurs $(r=0,55, p<0,001)$, entre les infractions sexuelles antérieures et les infractions sexuelles sans contact $(r=0,39, p<0,001)$, et entre l'agression sexuelle de victimes extrafamiliales et de victimes inconnues $(r=0,48, p<0,001)$.

\section{Nombre de facteurs retenus}

La solution factorielle basée sur les valeurs propres $(>1,0)$ était composée de trois facteurs, qui expliquaient $58,75 \%$ de la variance (voir tableau 4). Néanmoins, le graphique des éboulis (figure 1) suggérait qu’un 
quatrième facteur apportait une quantité non négligeable d'informations à la solution factorielle.

TAB LEA U 4

Valeurs propres et variance expliquée des facteurs

\begin{tabular}{|l|c|c|c|}
\hline Facteurs & Valeurs propres & $\begin{array}{c}\text { Pourcentage de } \\
\text { variance expliquée }\end{array}$ & $\begin{array}{c}\text { Pourcentage de } \\
\text { variance expliquée } \\
\text { cumulatif }\end{array}$ \\
\hline 1 $^{\text {er facteur }}$ & 2,03 & 22,50 & 22,50 \\
2 $^{\text {e facteur }}$ & 1,72 & 19,16 & 41,66 \\
3e facteur & $\mathbf{1 , 5 4}$ & $\mathbf{1 7 , 0 9}$ & $\mathbf{5 8 , 7 5}$ \\
4 $^{\text {facteur }}$ & 0,86 & 9,53 & 68,28 \\
5 $^{\text {facteur }}$ & 0,78 & 8,68 & 76,96 \\
6 $^{\text {facteur }}$ & 0,63 & 6,95 & 83,91 \\
$7^{\text {e facteur }}$ & 0,55 & 6,14 & 90,05 \\
8 $^{\text {facteur }}$ & 0,49 & 5,39 & 95,44 \\
$9^{\text {e }}$ facteur & 0,41 & 4,56 & 100,00 \\
\hline
\end{tabular}

F I G URE 1

Graphique des éboulis (scree test)

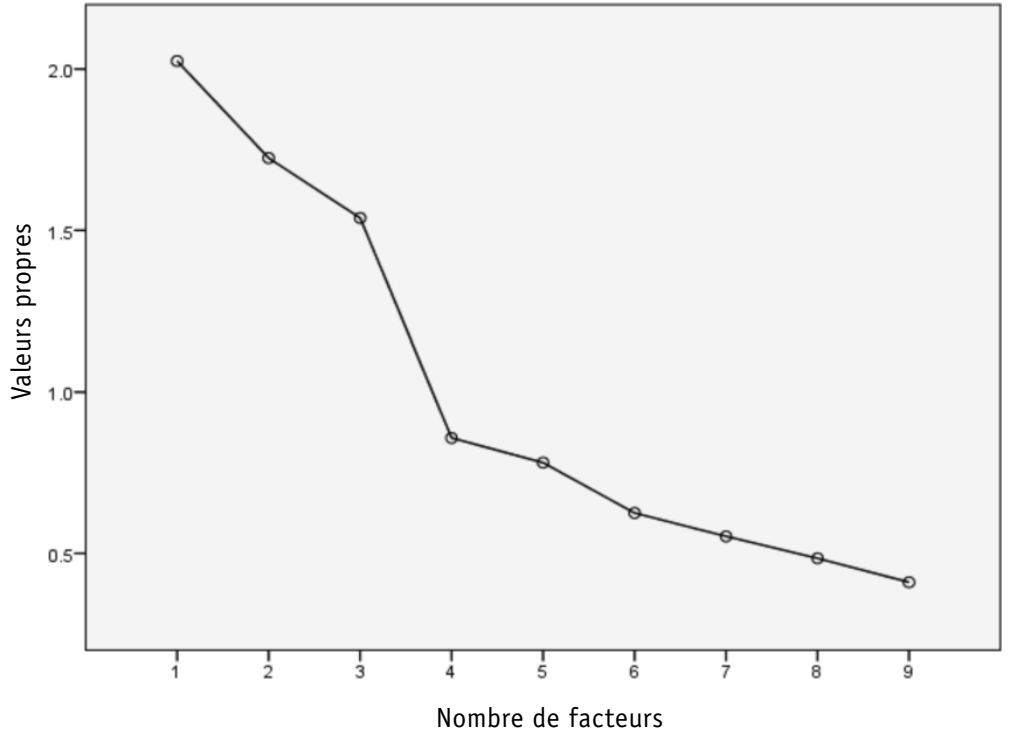

Une solution factorielle à quatre facteurs a alors été testée. L'ajout d'un facteur causa néanmoins l'apparition d'un artefact statistique (cas Heywood), dont l'explication la plus vraisemblable tient à un nombre 
de facteurs inadéquat (Tabachnick et Fidell, 2001). À ce titre, dans la solution à quatre facteurs, le dernier facteur n'était composé que d'un item, ce qui rendait son intérêt limité. La solution à quatre facteurs semblant créer davantage de problèmes qu'elle n'en résolvait, la solution à trois facteurs lui a été préférée.

\section{Solution factorielle}

La solution factorielle retenue présentait un indice d'adéquation qui était acceptable $(\mathrm{KMO}=0,60)$. Les saturations factorielles post-rotation sont présentées dans le tableau 5 . Le seuil de saturation pour l'inclusion d'un item dans un facteur était de 0,32 (Tabachnick et Fidell, 2001). L'item évaluant la violence non sexuelle dans le délit actuel ne saturait aucun facteur et fut conséquemment retiré de la solution factorielle. Aucun item ne saturait plus d'un facteur à la fois, reflétant une structure factorielle particulièrement nette.

TABLEA U 5

Analyse factorielle des items de la Statique-99R $(n=679)$

\begin{tabular}{|l|c|c|c|}
\hline \multirow{2}{*}{\multicolumn{1}{|c|}{ Items de la Statique-99R }} & \multicolumn{2}{c|}{ Saturations factorielles } \\
\cline { 2 - 4 } & $\begin{array}{c}\text { Facteur 1 } \\
\text { Délinquance } \\
\text { générale }\end{array}$ & $\begin{array}{c}\text { Facteur 2 } \\
\text { Jeune âge }\end{array}$ & $\begin{array}{c}\text { Facteur 3 } \\
\text { Délinquance } \\
\text { sexuelle }\end{array}$ \\
\hline Âge (score: -3 à +1) & $-0,01$ & $\mathbf{0 , 5 2}$ & $-0,29$ \\
Absence de cohabitation de plus de 2 ans & $-0,15$ & $\mathbf{0 , 5 0}$ & $-0,02$ \\
Violence non sexuelle dans antécédents & $\mathbf{0 , 7 7}$ & 0,02 & $-0,15$ \\
Infractions sexuelles antérieures (score: 0 à +3) & 0,18 & 0,06 & $\mathbf{0 , 6 6}$ \\
Prononcés de peine antérieurs & $\mathbf{0 , 7 3}$ & 0,00 & 0,12 \\
Infractions sexuelles sans contact & 0,02 & $-0,03$ & $\mathbf{0 , 5 4}$ \\
Victime sans lien de parenté & 0,12 & $\mathbf{0 , 6 2}$ & 0,19 \\
Victime inconnue & 0,10 & $\mathbf{0 , 5 9}$ & 0,09 \\
Victime de sexe masculin & $-0,15$ & 0,02 & $\mathbf{0 , 3 9}$ \\
\hline
\end{tabular}

$\mathrm{KMO}=0,60$

Le premier facteur était composé du nombre de prononcés de peines antérieurs et de la présence de violence non sexuelle dans les antécédents criminels, qui présentaient des saturations factorielles similaires (respectivement $\alpha=0,73$ et $\alpha=0,77$ ). Ce facteur a été nommé « délinquance générale», puisqu'il contenait un indicateur de délinquance non sexuelle et un indicateur de l'ampleur de la carrière criminelle - qu'elle soit sexuelle ou non. 
Le deuxième facteur était composé de l'âge $(\alpha=0,52)$, de l'absence de relation intime de plus de deux ans $(\alpha=0,50)$, et de l'agression sexuelle de victimes extrafamiliales $(\alpha=0,62)$ et inconnues $(\alpha=0,59)$. Il a tout simplement été nommé «jeune âge», puisque la manifestation conjointe des caractéristiques décrites par le facteur semblait le mieux expliquée par l'âge. En effet, un jeune délinquant sexuel a peu de chances d'être dans une relation intime de longue durée, et a ainsi rarement accès à des victimes intrafamiliales, qu'il s'agisse de sa partenaire ou de ses enfants. Il a donc plus de risques d'agresser sexuellement des victimes extrafamiliales ou inconnues, ayant par défaut moins accès à des victimes intrafamiliales qu'un délinquant plus âgé.

Le troisième facteur était composé des infractions sexuelles antérieures $(\alpha=0,66)$, des infractions sexuelles sans contact $(\alpha=0,542)$ et de l'agression sexuelle de victimes masculines $(\alpha=0,39)$. Puisque ce facteur était particulièrement bien représenté par les infractions sexuelles antérieures $(\alpha=0,66)$ et ne contenait que des items associés à la délinquance sexuelle, il a été nommé «délinquance sexuelle».

Les facteurs identifiés par l'analyse factorielle étaient somme toute très peu corrélés, malgré l'utilisation d'une méthode de rotation oblique (voir tableau 6). Cela indique qu'il n'y a pas de chevauchement des dimensions extraites.

TABLEAU 6

Corrélations entre les facteurs

\begin{tabular}{|l|c|c|c|}
\hline Facteurs & Délinquance générale & Jeune âge & Délinquance sexuelle \\
\hline Délinquance générale & 1,000 & - & - \\
Jeune âge & 0,079 & 1,000 & - \\
Délinquance sexuelle & 0,048 & 0,006 & 1,000 \\
\hline
\end{tabular}

\section{Discussion}

Les objectifs de la présente étude étaient d'identifier empiriquement les facteurs présents dans la Statique-99R, et d'en proposer une interprétation relativement aux dimensions psychologiques susceptibles de fournir un cadre conceptuel à un domaine largement considéré athéorique. Trois facteurs latents furent identifiés: la délinquance sexuelle, la délinquance générale et le jeune âge.

La nomenclature des facteurs a été faite avec prudence; un facteur latent n'est qu'un ensemble de variables fortement corrélées, dont la 
signification théorique et clinique relève de l'interprétation. Nous avons conséquemment préféré étiqueter nos facteurs en restant le plus près possible du contenu des items, afin de ne pas confondre les résultats empiriques et l'interprétation de ceux-ci.

\section{Interprétation des facteurs latents}

Délinquance sexuelle

Le facteur de délinquance sexuelle regroupait les infractions sexuelles antérieures, les infractions sexuelles sans contact et l'agression sexuelle de victimes masculines. Plusieurs auteurs ayant factorisé des outils actuariels pour délinquants sexuels ont obtenu un facteur similaire, qu'ils qualifiaient de déviance sexuelle (Roberts et al., 2002; Seto, 2005; Walters et al., 2009; Janka et al., 2010). L'association entre infractions sexuelles et déviance sexuelle n'est pas surprenante, dans la mesure où la majorité des fantaisies sexuelles déviantes, lorsqu'actualisées, sont génératrices de délinquance sexuelle ${ }^{5}$. En effet, les fantaisies sexuelles déviantes - au même titre que celles non déviantes - sont susceptibles d'influencer le comportement sexuel de l'individu (Eisenman, 1982; Leitenberg et Henning, 1995), le menant potentiellement à des comportements sexuels qui s'avéreront délictuels (Abel et Blanchard, 1974). De fait, il n'est pas étonnant que les intérêts sexuels déviants aient de forts liens statistiques avec une carrière criminelle hautement spécialisée en délits sexuels ${ }^{6}$ (Proulx et al., 2008), de même qu'avec la récidive sexuelle (Hanson et Bussière, 1998; Dempster et Hart, 2002; Doren, 2004; Hanson et Morton-Bourgon, 2004; Mann et al., 2010).

Il est toutefois possible que la délinquance sexuelle, telle que décrite par les items de notre facteur, relève de préoccupations sexuelles n'étant pas nécessairement déviantes. La notion de préoccupations sexuelles, en tant que dimension psychologique, a été abordée par Mann et al. (2010). Ils la définissaient comme un intérêt excessif et psychiquement envahissant pour la sexualité, qui, même en l'absence de fantaisies déviantes, peut mener à la délinquance sexuelle. En clinique, les délinquants sexuels font souvent état de pulsions sexuelles envahissantes qui, malgré qu'elles soient dirigées vers les femmes adultes, mènent à l'agression sexuelle. Par exemple, la recherche active de relations sexuelles peut altérer la per-

5. Par exemple: fantaisie d'agresser sexuellement une femme adulte de son entourage.

6. Telle que décrite par les items du facteur de délinquance sexuelle. 
ception du consentement chez l'autre, sans que des fantaisies violentes n'aient jamais été entretenues.

Ainsi, nous proposons que deux dimensions psychologiques, la déviance sexuelle et les préoccupations sexuelles, puissent être responsables de la manifestation des comportements décrits par notre facteur de délinquance sexuelle. Ces deux dimensions psychologiques bénéficient d'un bon appui empirique (Hanson et Morton-Bourgon, 2004; Mann et al., 2010), et sont bien représentées dans les instruments actuariels dynamiques de prédiction de la récidive sexuelle (Hanson et al., 2007).

\section{Délinquance générale}

Le facteur de délinquance générale incluait le nombre de prononcés de peine antérieurs et la présence de violence non sexuelle dans les antécédents criminels. Il fut nommé ainsi puisqu'il représentait essentiellement l'ampleur et le polymorphisme de la carrière criminelle générale. Un délinquant sexuel ayant un score élevé à ce facteur aura beaucoup de délits à son dossier, dont certains non sexuels et violents; cela fait davantage écho au profil du délinquant suractif (Cusson, 2005) qu'à celui de l'agresseur sexuel d'enfants hautement spécialisé7. Il faut néanmoins noter qu'une importante quantité de délits sexuels entraîne une cotation positive à l'un des items du facteur, soit l'item évaluant le nombre de prononcés de peines antérieurs. Malgré cela, le facteur de délinquance générale n'était aucunement corrélé avec le facteur de délinquance sexuelle, suggérant que le recoupement entre la délinquance sexuelle et l'item de prononcés de peines antérieurs n'était pas problématique.

L'item évaluant la violence non sexuelle commise lors du délit actuel était absent du facteur de délinquance générale. Cela est surprenant, dans la mesure où l'item l'évaluant lors des antécédents était le meilleur représentant du facteur. Il est toutefois possible que cette absence soit simplement due à une défaillance générale de l'item; en effet, l'item ne s'insérait dans aucune autre dimension de notre étude. De surcroît, il a déjà été identifié comme problématique par d'autres auteurs; c'est le seul item de la Statique-99R qui n'est pas associé à la récidive sexuelle (Helmus et Thornton, 2012). Si des recherches subséquentes corroborent son inadéquation, la suppression de cet item pourrait être envisagée.

7. Qui, nous en sommes conscients, est relativement peu commun (Harris et al., 2011). 
Au sujet des dimensions psychologiques de l'instrument; nous postulons que les assises psychologiques du facteur de délinquance générale sont les traits antisociaux et/ou la psychopathie. Les traits antisociaux sont définis par une indifférence vis-à-vis des normes sociales et des droits d'autrui, ainsi que par un comportement impulsif (American Psychiatric Association, 2000). La psychopathie, quant à elle, est caractérisée par un manque d'empathie et de remords, des émotions superficielles, de l'égocentrisme et de l'imposture (Cleckley, 1941 ; Hare, 2003). Ces deux troubles de personnalité se chevauchent, et sont fortement associés à une délinquance importante et violente (Hare, 1999; Hanson et MortonBourgon, 2004; Olver et Wong, 2006), telle que définie par les items de la dimension de délinquance générale.

Parallèlement, plusieurs recherches soulignent que la Psychopathy Checklist - Revised (PCL-R, Hare, 2003) - une échelle d'évaluation de la psychopathie - corrèle significativement avec la Statique-99, mais pas avec le RRASOR (Barbaree et al., 2001 ; Roberts et al., 2002; Seto et al., 2002; Doren, 2004). À ce sujet, la majorité des items de la Statique-99 couvrant la délinquance sexuelle proviennent du RRASOR; ils ont ainsi peu de chances de corréler avec la PCL-R. Il apparaît donc que les items responsables du lien entre la Statique-99 et la PCL-R se retrouvent dans les autres facteurs, soit ceux de délinquance générale ou de jeune âge. Puisque la psychopathie semble mieux représentée par l'activité criminelle décrite par le facteur de délinquance générale (Hare, 1999; Hanson et Morton-Bourgon, 2004; Olver et Wong, 2006) que par l'item du jeune âge, nous proposons que l'explication la plus vraisemblable de l'assise psychologique de la dimension de délinquance générale soit la personnalité antisociale/psychopathique.

\section{Jeune âge}

Le facteur de jeune âge regroupait (sans grande surprise) le jeune âge, l'absence de relation intime de plus de deux ans et l'agression sexuelle de victimes extrafamiliales/inconnues. Si certains auteurs ont interprété l'agression sexuelle de victimes éloignées relativement au détachement émotionnel (Roberts et al., 2002), nous avons préféré l'interpréter comme le simple effet du jeune âge. En effet, un jeune délinquant sexuel n'aura généralement pas disposé du temps nécessaire pour établir une relation intime à long terme, limitant ainsi son accès à des victimes intrafamiliales, qu'il s'agisse de sa partenaire ou de ses enfants. Dans la mesure 
où le choix de victimes extrafamiliales/inconnues découle de l'absence de relation intime - découlant à son tour du jeune âge -, il y a fort à parier que ce facteur fasse tout simplement état de la jeunesse du délinquant plutôt que d'un mécanisme psychologique latent.

Bien que le jeune âge ne soit ni une dimension psychologique, ni une cible d'intervention, sa présence dans l'instrument est tout de même pertinente, au regard du lien important existant entre l'âge et le crime, tant sexuel que non sexuel (Quételet, 1835; Hirschi et Gottfredson, 1983 ; Moffitt, 1993 ; Hanson, 2002 ; Sampson et Laub, 2003). Toutefois, nous ne prétendons pas que ce facteur soit l'expression comportementale d'une dimension psychologique.

\section{Conclusion}

La présente étude a permis d'extraire des facteurs latents de la Statique99R, et d'en proposer une interprétation relativement aux dimensions psychologiques susceptibles d'améliorer la compréhension qu'ont les évaluateurs d'un de leurs outils de travail. Deux des trois facteurs identifiés, soit la délinquance sexuelle et la délinquance générale, représentaient plutôt bien les deux dimensions psychologiques relevées précédemment dans l'instrument: la déviance sexuelle et les traits antisociaux (Doren, 2004; Brouillette-Alarie et Proulx, 2008; Nunes et Cortoni, 2008).

Bien que ces dimensions psychologiques ne soient certes pas les plus précises, ni les plus éclairantes en ce qui a trait aux objectifs cliniques, elles permettent d'illustrer qu'un instrument actuariel aussi mécanique que la Statique-99R pourrait ne pas fonctionner qu'en raison de la corrélation entre facteurs de risque et récidive. Bien que l'échelle ait été construite sur cette base, il y a tout de même lieu de se questionner sur les raisons pour lesquelles les facteurs de risque qu'elle contient prédisent efficacement la récidive (Mann et al., 2010); c'est là qu'une approche de facteurs latents telle que celle ici proposée se révèle pertinente.

Selon cette approche, la Statique-99R regroupe plutôt un ensemble de caractéristiques ou de comportements présentés par des individus dont les traits psychologiques les vulnérabilisent par rapport à une récidive potentielle. Bien que les items de l'échelle, soit des facteurs de risque statiques, n'aient pas de liens causaux avec la récidive, il n'en est pas de même pour les dimensions psychologiques qu'ils représentent. Ces dimensions, au même titre qu'un trouble de personnalité ou un schème de pensée, influencent la réponse comportementale fournie par un individu 
aux situations qu'il rencontre, et s'inscrivent dans une compréhension étiologique et situationnelle de la récidive. Il importe conséquemment que les études futures se concentrent davantage sur les dimensions psychologiques représentées par les instruments actuariels plutôt que sur les outils en soi.

\section{Limites et recherches futures}

Les propositions théoriques de la présente étude, bien que novatrices, doivent être considérées avec précaution, vu les tenants et les aboutissants de l'analyse factorielle. D'une part, l'analyse factorielle est conçue pour traiter des variables continues se distribuant normalement, étant basée sur une matrice de corrélations régulières. Puisque les variables de notre étude (les items de la Statique-99R) sont principalement dichotomiques, une matrice de corrélations tétrachoriques aurait dû être employée (Kubinger, 2003). Toutefois, la procédure d'analyse factorielle de SPSS 20 ne permet pas l'utilisation d'une telle matrice, influençant potentiellement la structure factorielle en résultant. Bien que certains auteurs affirment qu'il y a peu de différences entre l'utilisation de matrices de corrélations régulières et tétrachoriques (Percy, 1976), les recherches futures sur le sujet devraient se pencher sur l'influence respective de chacune de ces matrices.

D'autre part, l'analyse factorielle postule que si certaines variables (comportements) sont fortement corrélées, c'est parce qu'un facteur latent cause la manifestation conjointe de ces comportements. Toutefois, comme il a par ailleurs été observé dans cette étude, certaines variables peuvent corréler (par exemple: jeune âge et absence de relation intime de plus de deux ans) sans qu'une entité clinique ne sous-tende cette corrélation (Ruscio et al., 2006). Il en va de même pour l'ensemble de nos facteurs: nous ne disposons d'aucune garantie qu'ils soient réellement des dimensions psychologiques. Parallèlement, la nomenclature des dimensions psychologiques relève de l'interprétation des auteurs, et non pas des données empiriques. Il est donc impératif de garder en tête que les interprétations proposées sont spéculatives, et doivent faire l'objet d'études de validité de construit avant d'être intégrées à une quelconque pratique clinique. Un tel devis de recherche permettrait de déterminer si les facteurs latents extraits par la présente étude sont des construits psychologiques réels, cohérents et pertinents pour la pratique correctionnelle. Nous espérons que de telles recherches seront entreprises dans le futur. 


\section{Références}

Abel, G. G., \& Blanchard, E. E. (1974). The role of fantasy in the treatment of sexual deviation. Archives of General Psychiatry, 30, 467-475.

American Psychiatric Association. (2000). Diagnostic and statistical manual of mental disorders (4 ${ }^{\text {th }}$ Ed., Text Revision). Washington: Author.

Archer, R. P., Buffington-Vollum, J. K., Stredny, R. V., \& Handel, R. W. (2006). A survey of psychological test use patterns among forensic psychologists. Journal of Personality Assessment, 87, 84-94.

Babchishin, K. M., Hanson, R. K., \& Helmus, L. (2011). The RRASOR, Static$99 R$, and Static-2002R all add incrementally to the prediction of recidivism among sex offenders (User Report No. 2011-02). Ottawa: Public Safety Canada.

Barbaree, H. E., Seto, M. C., Langton, C. M., \& Peacock, E. J. (2001). Evaluating the predictive accuracy of six risk assessment instruments for adult sex offenders. Criminal Justice and Behavior, 28, 490-521.

Beech, A. R., Friendship, C., Erikson, M., \& Hanson, R. K. (2002). The relationship between static and dynamic risk factors and reconviction in a sample of UK child abusers. Sexual Abuse: A Journal of Research and Treatment, 14, 155-167.

Beech, A. R., \& Ward, T. (2004). The integration of etiology and risk in sexual offenders: A theoretical framework. Aggression and Violent Behavior, 10, 31-63.

Brouillette-Alarie, S., \& Proulx, J. (2008). Predictive and convergent validity of phallometric assessment in relation to sexual recidivism risk. Conférence présentée au $27^{\mathrm{e}}$ congrès de 1'Association for the Treatment of Sexual Abusers, Atlanta, GA.

Cleckley, H. (1941). The mask of sanity. St. Louis: Mosby.

Cortoni, F. (2009). Factors associated with sexual recidivism. In A. R. Beech, L. A. Craig, \& K. D. Browne (eds.), Assessment and Treatment of Sex Offenders: $A$ Handbook (39-52). West Sussex: John Wiley \& Sons Ltd.

Costello, A. B., \& Osborne, J. W. (2005). Best practices in exploratory factor analysis: Four recommendations for getting the most from your analysis. Practical Assessment Research \& Evaluation, 10 (7), 1-9.

Cusson, M. (2005). La délinquance, une vie choisie. Entre plaisir et crime. Montréal: Éditions HMH Hurtubise, Cahiers du Québec, Collection Droit et criminologie.

Dawes, R. M., Faust, D., \& Meehl, P. E. (1989). Clinical versus actuarial judgment. Science, 243, 1668-1774.

Dempster, R. J., \& Hart, S. D. (2002). The relative utility of fixed and variable risk factors in discriminating sexual recidivists and nonrecidivists. Sexual Abuse: A Journal of Research and Treatment, 14 (2), 121-138.

Doren, D. M. (2004). Towards a multidimensional model for sexual recidivism risk. Journal of Interpersonal Violence, 19, 835-856. 
Eisenman, R. (1982). Sexual behavior as related to sex fantasies and experimental manipulation of authoritarianism and creativity. Journal of Personality and Social Psychology, 43, 853-860.

Epperson, D. L., Kaul, J. D., Huot, S. J., Hesselton, D., Alexander, W., \& Goldman, R. (1998). Minnesota Sex Offender Screening Tool - Revised (MnSOST-R). St. Paul: Minnesota Department of Corrections.

Gardner, W., Lidz, C. W., Mulvey, E. P., \& Shaw, E. C. (1996). Clinical versus actuarial predictions of violence in patients with mental illnesses. Journal of Consulting and Clinical Psychology, 64, 602-609.

Gendreau, P., Little, T., \& Goggin, C. (1996). A meta-analysis of the predictors of adult offender recidivism: What works! Criminology, 34, 575-607.

Grove, W. M., Zald, D. H., Hallberg, A. M., Lebow, B., Snitz, E. \& Nelson, C. (2000). Clinical versus mechanical prediction: A meta-analysis. Psychological Assessment, 12, 19-30.

Grubin, D. (1998). Sex offending against children: Understanding the risk. Police Research Series Paper 99. London: Policing and Reducing Crime Unit Home Office.

Guay, J.-P. (2006). Prédiction actuarielle et prédiction clinique: le dernier souffle d'une pratique traditionnelle. Revue internationale de criminologie et de police technique et scientifique, 59, 149-164.

Hanson, R. K. (1997). The development of a brief actuarial risk scale for sexual offense recidivism (User Report No. 1997-04). Ottawa: Department of the Solicitor General of Canada.

Hanson, R. K. (2002). Recidivism and age: Follow-up data from 4,673 sexual offenders. Journal of Interpersonal Violence, 17, 1046-1062.

Hanson, R. K., \& Bussière, M. T. (1996). Predictors of sexual offender recidivism: A meta-analysis (User Report No. 1996-04). Ottawa: Department of the Solicitor General Canada.

Hanson, R. K., \& Harris, A. (1998). Dynamic Predictors of Sexual Recidivism (User Report No. 1998-01). Ottawa: Department of the Solicitor General Canada.

Hanson, R. K., Harris, A. J. R., Scott, T.-L., \& Helmus, L. (2007). Assessing the risk of sexual offenders on community supervision: The Dynamic Supervision Project (User Report No. 2007-05). Ottawa: Public Safety Canada.

Hanson, R. K., \& Morton-Bourgon, K. E. (2004). Predictors of sexual recidivism: An updated meta-analysis (User Report No. 2004-02). Ottawa: Public Safety and Emergency Preparedness Canada.

Hanson, R. K., \& Morton-Bourgon, K. E. (2009). The accuracy of recidivism risk assessments for sexual offenders: A meta-analysis of 118 prediction studies. Psychological Assessment, 21 (1), 1-21.

Hanson, R. K., \& Thornton, D. (2000). Improving risk assessments for sex offenders: A comparison of three actuarial scales. Law and Human Behavior, 24 (1), 119-136.

Hare, R. D. (1999). Psychopathy as a risk factor for violence. Psychiatric Quarterly, 70 (3), 181-197. 
Hare, R. D. (2003). The Psychopathy Checklist-Revised (2 ${ }^{\text {nd }}$ Ed.). Toronto: MultiHealth Systems.

Harris, D. A., Knight. R. A., Smallbone, S., \& Dennison, S. (2011). Post-release specialization and versatility in sexual offenders referred for civil commitment. Sexual Abuse: Journal of Research and Treatment, 23 (2), 243-259.

Harris, G. T., Rice, M. E., \& Quinsey, V. L. (1993). Violent recidivism of mentally disordered offenders: The development of a statistical prediction instrument. Criminal Justice and Behavior, 20, 315-335.

Helmus, L. (2009). Re-norming Static-99 recidivism estimates: Exploring base rate variability across sex offender samples (Master's thesis). Consulté le 27 septembre 2012, http://static99.org/pdfdocs/helmus2009-09static-99normsmathesis. pdf

Helmus, L., \& Thornton, D. (2012). Performance of individual items of Static99R and Static-2002R. Conférence présentée au $31^{\mathrm{e}}$ congrès de l'Association for the Treatment of Sexual Abusers, Denver, CO.

Hirschi, T., \& Gottfredson, M. (1983). Age and the explanation of crime. American Journal of Sociology, 89, 552-584.

Jackson, R. L., \& Hess, D. T. (2007). Evaluation for civil commitment of sex offenders: A survey of experts. Sexual Abuse: A Journal of Research and Treatment, 19, 409-448.

Janka, C., Gallasch-Nemitz, F., \& Dahle, K.-P. (2011). Zur Altersabhängigkeit von Risikovariablen bei Sexualdelinquenz. Forens Psychiatr Psychol Kriminol, $5,37-44$.

Jöreskog, K. G. (2003). Factor analysis by MINRES. Consulté le 27 septembre 2012, http://www.ssicentral.com/lisrel/techdocs/minres.pdf

Knight, R. A., \& Thornton, D. (2007). Evaluating and improving risk assessment schemes for sexual recidivism: A long-term follow-up of convicted sexual offenders (Document No. 217618). Washington: U.S. Department of Justice.

Kubinger, K. D. (2003). On artificial results due to using factor analysis for dichotomous variables. Psychology Science, 45, 106-110.

Leitenberg, H., \& Henning, K. (1995). Sexual fantasy. Psychological Bulletin, 117 (3), 469-496.

Mann, R. E., Hanson, R. K., \& Thornton, D. (2010). Assessing risk for sexual recidivism: Some proposals on the nature of psychologically meaningful risk factors. Sexual Abuse: A Journal of Research and Treatment, 22 (2), 191-217.

McGrath, R. J., Cumming, G. F., Burchard, B. L., Zeoli, S., \& Ellerby, L. (2010). Current practices and emerging trends in sexual abuser management: The safer society 2009 North American survey. Brandon, VT: Safer Society.

Meehl, P. E. (1954). Clinical versus statistical prediction: A theoretical analysis and a review of the evidence. Minneapolis: University of Minnesota Press.

Moffitt, T. E. (1993). Adolescence-limited and life-course-persistent antisocial behavior: A developmental taxonomy. Psychological Review, 100, 674-701.

Mossman, D. (1994). Assessing predictions of violence: being accurate about accuracy. Journal of Consulting and Clinical Psychology, 62, 783-792. 
Nunes, K., \& Cortoni, F. (2008). Dropout from sex-offender treatment and dimensions of risk of sexual recidivism. Criminal Justice and Behavior, 35, 24-33.

Olver, M. E., \& Wong, S. C. P. (2006). Psychopathy, sexual deviance, and recidivism among sex offenders. Sexual Abuse: A Journal of Research and Treatment, $18(1)$, 65-82.

Parent, G., Guay, J.-P., \& Knight, R. A. (2011). An assessment of long-term risk of recidivism by adult sex offenders: One size doesn't fit all. Criminal Justice and Behavior, 38, 188-209.

Percy, L. (1976). An argument in support of ordinary factor analysis of dichotomous variables. Advances in Consumer Research, 3, 143-148.

Pham, T.-H., \& Ducro, C. (2008). Évaluation du risque de récidive en Belgique francophone: données préliminaires d'analyse factorielle de la «Sex Offender Recidivism Appraisal Guide» (SORAG) et de la Statique-99. Annales Médico-Psychologiques, 166, 575-579.

Proulx, J., Lussier, P., Ouimet, M., \& Boutin, S. (2008). Criminal careers of four types of sexual aggressors. In B. Schwartz (ed.), Sex Offenders (Vol. 6, p. 1-21). New York: Civic Research Institute.

Quételet, A. (1835). Sur l'bomme et le développement de ses facultés, ou Essai de physique sociale. Paris: Bachelier, Imprimeur-libraire, Quai des Augustins, $\mathrm{n}^{\circ} 55$.

Quinsey, V. L., Harris, G. T., Rice, M. E., \& Cormier, C. A. (2006). Violent offenders: Appraising and managing risk (2 ${ }^{\text {nd }}$ Ed.). Washington: American Psychological Association.

Roberts, C. F., Doren, D. M., \& Thornton, D. (2002). Dimensions associated with assessments of sex offender recidivism risk. Criminal Justice and Behavior, 29, 569-589.

Ruscio, J., Haslam, N., \& Ruscio, A. M. (2006). Introduction to the taxometric method: A practical guide. Mahwah: Lawrence Erlbaum Associates.

Sampson, R. J., \& Laub, J. H. (2003). Life-course desisters? Trajectories of crime among delinquent boys followed to age 70. Criminology, 41, 301-339.

Seto, M. C. (2005). Is more better? Combining actuarial risk scales to predict recidivism among adult sex offenders. Psychological Assessment, 17 (2), 156167.

Seto, M. C., Barbaree, H. E., \& Langton, C. M. (2002). How should we interpret the results of multiple actuarial risk assessment instruments? Conférence présentée au $21^{\mathrm{e}}$ congrès annuel de l'Association for the Treatment of Sexual Abusers, Montréal, QC.

Tabachnick, B. G., \& Fidell, L. S. (2001). Using multivariate statistics (4 ${ }^{\text {th }}$ Ed.). New York: Harper Collins Publishers.

Thornton, D. (2002). Constructing and testing a framework for dynamic risk assessment. Sexual Abuse: A Journal of Research and Treatment, 14, 137-151.

Walters, G. D., Deming, A., \& Elliott, W. N. (2009). Assessing criminal thinking in male sex offenders with the psychological inventory of criminal thinking styles. Criminal Justice and Behavior, 36, 1025-1037. 
ABSTRACT - The aim of the current paper is to identify dimensions of the Static-99R, the most commonly used sex-offender actuarial scale. Exploratory factor analysis extracted three dimensions from the scale: sexual criminality, general criminality and young age. An interpretation of the psychological meaning of these dimensions was suggested: sexual criminality as an indicator of either sexual deviance or sexual preoccupations, and general criminality as an indicator of antisocial traits and/or psychopathy.

KEYWORDS - Sex offenders, static risk factors, dynamic risk factors, Static-99, factor analysis, psychologically meaningful risk factors.

RESUMEN - Una critica comúnmente formulada hacia las herramientas estadísticas actuariales consiste en su incapacidad para aclarar la intervención, debido sus bases no teóricas. En el marco de un esfuerzo por matizar dicha crítica, el presente estudio ha intentado identificar las construcciones teóricas presentes en Statistique-99R, la escala actuarial más utilizada en lo que a predicción de la reincidencia de los delincuentes sexuales se refiere. Un análisis factorial exploratorio de los elementos de dicho instrumento extrajo tres factores: la delincuencia sexual, la delincuencia general y la edad joven. Una interpretación de estos factores en términos psicológicos ha sido propuesta: la delincuencia sexual como índice de desviación sexual o de inquietudes sexuales, y la delincuencia general como indicador de rasgos antisociales o de psicopatía. Los límites y las implicaciones para futuras investigaciones son aquí abordados.

PALABRAS CLAVE - Agresores sexuales, factores de riesgo estadísticos, factores de riesgo dinámicos, Statistique-99R, análisis factorial. 